\title{
El adjetivo en el análisis de errores basado en la autorregulación como tratamiento del error
}

\section{The adjective in error analysis based on self-regulatory practices as error treatment Jesús E. Fong-Flores ${ }^{a}$}

\begin{abstract}
:
Within language learning contexts, error analysis represents a tool that enables teachers to identify causes in target language performance. By means of a corpus built from authentic written texts produced by students of French as a Foreign Language, this contribution analyses errors using the etiological criteria. This study, which has a mixed-methods research design and a descriptive scope, aims to identify errors in the use of adjectives and their gender and number agreement in French. The corpus is made of 188 written productions in French level A1, as well as 171 written productions in level A2. The texts were produced by students pursuing a degree in foreign language teaching. The foregoing is considered a variable within this study that collects both quantitative and qualitative data as it observes recurrences of the error while making an in-depth analysis to try to determine the causes behind it. The results show the importance of error identification in language teaching and how it can be addressed through self-regulatory practices.
\end{abstract}

Keywords:

Errors, tipology, error treatment, french as foreign language

\section{Resumen:}

El análisis de errores dentro de los contextos formales de aprendizaje representa un medio mediante el cual identificamos cau sas y razones de desempeño en la lengua meta. Desde la perspectiva del criterio etiológico, este estudio analiza mediante un corpus de estudiantes auténtico, la producción del texto escrito en francés lengua extranjera. El objetivo general es reconocer la presencia de errores en el adjetivo y su concordancia entre género y número. El corpus se compone 188 producciones escritas en el nivel A1 y 171 en nivel A2 donde se hace hincapié en la descripción del error basado en un enfoque mixto de alcance descriptivo. Los tex tos fueron escritos por estudiantes de francés lengua extranjera pertenecientes al centro de lenguas que, específicamente, siguen una formación para la enseñanza de lenguas; considerando lo anterior una variable para el estudio. Se considera un estudio cuantitativo al observar recurrencias del error y cualitativo al hacer un análisis a profundidad que determina las causas del error. Los resultados muestran la importancia del reconocimiento del error y cómo éste puede ser tratado mediante prácticas de autorregulación.

Palabras Clave:

Errores, tipología, tratamiento del error, francés lengua extranjera 


\section{Introducción}

Basados en la conformación de nuestro corpus escrito de estudiantes de francés lengua extranjera (FLE), partimos a la búsqueda de las representaciones del error en el adjetivo y la concordancia entre género y número. Este acercamiento al error, dentro de los contextos formales de aprendizaje, puede abordarse desde distintas líneas de investigación que delimitarán un proceso único. Entendemos que, en cuanto al error, existen diversos criterios y tipologías mediante los cuales podemos clasificarlo; siendo nuestra intención la evaluación y al tratamiento del error en el aula de lengua extranjera (LE).

En una producción escrita desde su interpretación como producto, es el profesorado quien analiza la calidad de la producción del texto escrito, quien identifica la presencia de elementos que reflejan una competencia en la lengua meta, y que en algunos casos reconoce errores. Pero la cuestión es razonar qué nos indican esos errores pues en ocasiones son condicionados por distintos factores dependientes del contex to de aprendizaje. Algunos de los errores podríamos justificarlos por que surgen de un recurso estratégico por parte del estudiante de LE, u otra razón puede surgir en función a otras lenguas que forman parte de su repertorio lingüístico.

En este documento expresamos que la presencia de estas lenguas próximas, o vistas como esas con las que tienen contacto los hablantes, influyen de cierta forma en el error. No obstante, debemos puntualizar que la presencia del error desde la interferencia lingüística no es la única condición; por el contrario, debemos atribuir a otras tipologías también presentes como los errores intralingüísticos, causados por desviaciones o generalizaciones.

Por lo que refiere a la transferencia negativa, ésta surge de la acción de recurrir a las lenguas próximas con el fin de estratégicamente comunicar en una lengua meta. En algunos casos, podemos ver a este movimiento estratégico como un recurso conveniente para el estudiante, pero, en otros casos no resulta favorable. Pensamos en el mismo tenor, que la esencia del error se debe a un aprendizaje aún en tránsito el cual detallaremos posteriormente.

El objetivo principal de nuestra investigación es reconocer la presencia de errores en el adjetivo y su concordancia desde el criterio etiológico. Por lo que este acercamiento al error lo abord amos en dos tipologías medulares contempladas en el tratamiento del error dentro de nuestro corpus de FLE el cual ubica: errores interlinguales y errores intralinguales.

Para hacer esto posible, los datos tratados surgen de nuestro corpus de estudiantes compuesto por producciones escritas en francés lengua extranjera en el nivel A1 y A2. Pero, antes de describir el estudio ponemos sobre la mesa el por qué es importante entender el error y no sólo identificarlo como un elemento generalizado. En primera instancia, bajo la idea de que el error representa un recurso que nos permite diagnosticar las competencias en la lengua meta por parte del estudiante. En segunda, notamos que a través de la identificación de los distintos errores es posible construir propuestas didácticas y pedagógicas que permitan un tratamiento contextualizado del error en el aula. Siendo nuestra investigación una aportación al área de FLE y al campo del tratamiento del error el cual desde tiempo atrás contempla distintos acercamientos: lo cognitivo (Dewaele, 1998), la tipología del error (Cenoz, 2001), el estudio de la interlengua (De Angelis y Selinker, 2001; Odlin, 1989), la frecuencia de errores (Granger, 2003; Hammarberg, 2001), y esos de tipo intralinguales (Touchie, 1986; Vázquez, 1999).

En línea con este debate, el análisis lingüístico basado en nuestro corpus de estudiantes hispanohablantes de Francés Lengua Extranjera permite reconocer que, si existen errores en el adjetivo existe también una explicación que se sustenta bajo una tipología específica. En nuestro contexto institucional de Facultad de Idiomas de la UABC, como profesores formadores en el campo de FLE u otras lenguas extranjeras, debemos plantear que el estudiante de lengua busca ser un miembro activo en su aprendizaje. Es decir que buscará comunicar a pesar de los errores que pueda cometer que, en todo caso, es una negociación entre sus competencias, conjugadas con las nuevas competencias que deberá concientizar. A su vez, esta concientización es dependiente del cómo valoramos nuevas rutas u opciones hacia el tratamiento del error en el aula de FLE.

\section{Marco teórico}

En este apartado se expresa un acercamiento al análisis de errores aplicado a los contextos formales de aprendizaje, con especial énfasis en detallar sus principios. Posterior, se caracteriza el criterio y la tipología del error que nos orienta hacia su categorización y nos dirige a la autorregulación como recurso para el tratamiento del error.

\section{Desde el análisis al error en el aula}

El punto de partida se centra en expresar que la lengua es un recurso de comunicación, el cual se compone de normas y reglas específicas que deberán respetarse por parte del aprendiente. En un momento donde el estudiante no tiene una conciencia de y por la lengua, surgen posibles barreras o errores que se condicionan por diversos factores; de ahí que la presencia de errores demanda un análisis estructurado el cual expresamos a continuación. El Análisis de Errores (AE) como una corriente de investigación permitió desde tiempo atrás una perspectiva distinta hacia la interpretación del error. Lo anterior, hizo posible establecer criterios y tipologías que se situaron específicamente en el campo de la lingüística aplicada a la enseñanza de lenguas (James, 1998; Lyster, 2001). 
En cuanto al Análisis de Errores dejemos claro que el interés principal se centra en determinar causas y razones que permiten una comprensión del uso de la lengua (Alexopoulou, 2005; Corder, 1983; Fernández, 1997; Santos, 1993; Selinker, 1972; Vázquez, 1991). Desde luego, el Análisis de Errores como propuesta hacia la comprensión de escenarios de aprendizaje ha obtenido una revaloración sobre todo desde los nuevos enfoques que han surgido, por mencionar esos del corpus (Ferreira et al.,2014; Granger, 2009). Se trata en lo particular, de un análisis estructurado que se construye a partir de ciertos movimientos o pasos que debemos delimitar bajo la intención del investigador. Ellis (1986) parte del hecho que hacia el análisis del error conviene partir de la siguiente manera: la conformación de un corpus, la identificación de errores, su clasificación y explicación. Por lo que refiere al análisis, compartimos que éste es dependiente de los intereses propios de la investigación pues su alcance será razón de los datos que se buscan obtener o socializar, considerando que a partir del corpus de estudiantes la información se abre a diversos enfoques para su estudio.

Dentro de nuestro análisis queremos destacar que la construcción de un corpus autentico para su posterior interpretación permite un acercamiento único a los escenarios de aprendizaje, sobre todo bajo la idea de que ningún contexto es igual. Pendar y Chapelle (2008) precisan que es a través de la conformación de un corpus que resulta factible tener datos auténticos para ser procesados.

Tras otorgar un valor general al Análisis de Errores, trazamos que el error brinda un punto de identificación del nivel de desempeño de la lengua por parte del estudiante (Ellis, 1985). El autor expone que merece la pena que reconozcamos al error como una fuente de información valiosa que permite diferentes interpretaciones. De tal modo que observemos al error como algo distinto y que provee de un mejor entendimiento del escenario de aprendizaje. Donde por mencionar, si existen errores en el adjetivo y la concordancia entendemos que existe un por qué y también un para qué. Un por qué bajo la idea que surgen factores que influyen en el desarrollo de la lengua: las lenguas próximas, la cultura, la exposición a la lengua, y los recursos didácticos. Y el para qué, donde el error para el estudiante tanto como para el profesor sea un elemento que propicie la reflexión y la autoevaluación.

Así pues, en la producción escrita y en cualquier competencia de la lengua se debe insistir que el error estará siempre presente. Pero que su interpretación demanda de una postura distinta, donde se comprenda, analice, y sensibilice qué posición (como formadores) buscamos otorgar al error. Más claro se hace en Scott, Freeman y Patel (2002) tras indicar que la evaluación del aprendizaje es un proceso complejo. Sin embargo, nos recuerdan que debemos de hablar de un aprendizaje en tránsito o dinámico donde existen diversos conocimientos por descubrir y sobre todo de reflexionar.
De esta forma, planteamos que un elemento funcional se sitúa en reconocer las características que posee un error y sus variaciones. En nuestra aula de FLE, destacamos la importancia que tiene entender el error y que éste puede verse como un error temporal, desde el aprendizaje aún en camino, o ese error que tiende a mantenerse dentro del trayecto formativo y en algunos casos hacia una fosilización. De aquí la importancia de hacer distinciones de lo que figura como error (Ellis, 1997).

Bajo el foco de esta investigación planteamos, entonces, que el error se atribuye a una falta de concientización y sensibilización metalinguiística que formará parte de las estrategias que considera el estudiante para comunicar. Al sintetizar al error desde su análisis, Besse y Porquier (1984, p.207) proponen:

L'analyse des erreurs doit avoir un double objectif: l'objectif théorique afin de mieux comprendre le processus d'apprentissage d'une langue étrangère; l'objectif pratique afin d'améliorer l'enseignement de cette langue. Ces deux objectifs s'articulant l'un à l'autre permettent une meilleure compréhension de processus d'apprentissage et contribuent à la conception de principes et de pratiques d'enseignement mieux appropriés, où sont reconnus et acceptés le statut et la signification des erreurs.

En este orden, vemos que este tipo de estudios basados en la identificación y/o análisis de errores permiten una dirección al proceso de enseñanza y aprendizaje. Así seremos capaces de definir que el error representa un elemento inevitable:

"l'erreur est non seulement inévitable, mais normal et nécessaire, constituant un indice et un moyen d'apprentissage. On n'apprend pas sans faire d'erreurs et les erreurs servent à apprendre" (Porquier, 1977, p.28).

Al sintetizar dicha información establecemos en nuestra investigación que el análisis del error, en particular desde el adjetivo y su concordancia, no sólo pone en evidencia las causas también permite poner en contexto las implicaciones didácticas y pedagógicas que deberán formar parte del quehacer profesional docente.

\section{El criterio etiológico y la tipología del error}

Bajo la intención de este estudio ponemos la mirada hacia el criterio etiológico, el cual consiste en determinar las causas y el origen del error basados en una tipología específica (Alexopoulou, 2006; De Alba, 2009). Hacia la clasificación del error, Gómez y Cabrera (2016), comparten que los criterios lingüísticos y etiológicos han sido más frecuentes en el $\mathrm{AE}$.

Pasando a la tipología la cual detallaremos, observamos que en nuestra aula de FLE existen dos tipos de errores recurrentes: 1) errores que se asocian a las lenguas próximas del estudiante en formación, 2) errores que se reconocen como desviaciones o generalizaciones; priorizando 
este último dentro de este texto. La presencia de estos errores se ubica, como Han y Odlin (2006) expresan, en cualquier elemento lingüístico. Antes de entrar completamente a la tipología del error, reconocemos que la producción escrita es un conjunto de elementos linguiísticos, palabras, frases y oraciones cuya intención es transmitir un mensaje. Siendo medular, que el estudiante sea consciente de esos recursos linguiísticos que le permitirán esta comunicación de la cual se habló a inicios de este documento.

En el orden que nos corresponde, pasamos a la descripción de qué refiere cada error desde su tipología. El error interlingual, visto tiempo atrás en Selinker (1972) se muestra como la influencia lingüística de otras lenguas que son intentos de asociación temprana. Griffin (2005) establecía que los errores provocados por la interlengua refieren a una aproximación a las reglas que surgen del repertorio lingüístico de otras lenguas. Otros autores, mencionan que es asociada como consecuencia de un sistema aproximativo (Nemser, 1971).

Basado en los elementos expuestos, es necesario situar a la interlengua como un referente transitivo y dependiente del contexto. En nuestro caso, cobra bastante relevancia considerar el escenario de aprendizaje debido a la influencia de otras lenguas hacia el aprendizaje del francés como lengua extranjera o lengua meta. Yule (1996) sostiene que en la producción de una lengua meta existe un estadio que contempla características linguísticas de una lengua materna y esa segunda. Baralo (1999) presenta que la interlengua es una estructura construida por el hablante a partir de la asociación de sistemas lingüísticos. Dentro de este espacio será posible observar que los mecanismos lingüísticos de las lenguas próximas tienden a figurar en la lengua meta. Antes de cerrar este párrafo, concretamos que los errores interlinguales son a razón de la influencia de una lengua materna u otras que presentan variaciones hacia el nuevo compuesto lingüístico (Brown, 2006). Sin olvidar que esta tipología interlingual, insistimos, se condiciona por el contexto y la exposición a otras lenguas.

La otra parte que atiende nuestro acercamiento tipológico, nos dirige ahora al error intralingual; elemento primordial para este estudio. Richards (1974) citado en Alexopoulu (2010) establece que la noción intralingüística es el resultado de un conflicto interno de reglas. La autora muestra que este error intralingüístico surge a razón de un sistema de tipo simplificado o, por generalizaciones a las reglas que rige la lengua meta. Lo mismo, descrito en Touchie (1986) quien estableció que los errores desde esta tipología se centraban principalmente en lo siguiente: errores de simplificación, de generalizaciones, u omisión. Lantolf (2006), en resumen, nos presenta que los errores interlinguales derivan de una transferencia negativa de las lenguas próximas del individuo, y por otra, reconoce a los errores intralinguales como desviaciones a reglas y normas.

Es así que, bajo los intereses propios de nuestro estudio vemos que estas dos tipologías enmarcan una intención particular. Por un lado, donde el estudiante tiende generalizar cuestiones de género en el adjetivo: es decir masculino y femenino sin analizar al sustantivo. Por otra, de no considerar el aspecto cuantificable donde el adjetivo se condiciona por el singular o plural. En cuanto al error por transferencia de otras lenguas vemos claro que el aprendiz parte del conocimiento previo y busca asociar con el fin de comunicar. Es decir, piensa en cómo produce el adjetivo en su lengua materna $\mathrm{u}$ otra próxima dentro de su repertorio lingüístico. Es de ahí que las diversas tipologías descritas proveen un acercamiento diferente hacia el tratamiento del error.

\section{EI tratamiento del error en el aula de FLE desde la autorregulación}

Desde la función que expresa el criterio y tipología antes descritos, consideramos la necesidad de plantear cómo es posible tener un acercamiento a la evaluación y tratamiento del error. En definitiva, partimos del hecho que cualquier error es evaluable y medible pero también tratable. En este sentido, los errores en la producción de una lengua extranjera son justificables desde el entendido que el estudiante está en un proceso de aprendizaje.

En este momento o tránsito es donde el formador debe ver distintas rutas mediadoras hacia el tratamiento del error. Por lo anterior optamos por compartir la aportación que hace Alexoupoulou (2005), quien establece la importancia de sensibilizar al estudiante en los fenómenos lingüísticos, a reconocer sus errores, y autocorregirse. Esto último resulta complejo por que es dependiente de las características y estrategias de los estudiantes.

En relación con lo anterior, abonamos que uno de los elementos que valdría exponer dentro de esta propuesta es la autorregulación. Se define a la autorregulación como el proceso que permite al individuo tener una reflexión de su propio aprendizaje. En donde sea capaz de autoevaluarse y comprend a qué elementos requerirá para cumplir con una meta específica dentro del curso de LE. Por lo que tendríamos que expresar que la autorregulación no resulta sólo como un producto sino como un proceso de seguimiento y evaluación diversificada e integral. Destacando que la evaluación (sumativa o formativa) debe dirigirse desde una socialización que propicie una percepción distinta sobre el cómo es evaluado el estudiante y cómo interpreta su evaluación. El reto va, desde luego, en las maneras que el docente pudiera fomentar estas prácticas de autorregulación, dejando claro que este proceso requerirá de diversos elementos como: la cognición, la motivación y la apreciación hacia la lengua meta (Pintrich, 2000). Agregamos, que ésta considera otros elementos como la metacognición, las motivaciones, y conductas en el proceso de aprendizaje 
(Zimmerman, 2000). En sí, destacamos que la autorregulación propicia una mayor responsabilidad y análisis por el aprendizaje (Aregu, 2013).

Ciertamente, por que resulta justo mencionarlo, el factor de la responsabilidad del estudiante por su aprendizaje es complejo; pues en ocasiones está fuera del quehacer docente. Sin embargo, es precisamente hacia la construcción e implementación de prácticas autorreguladoras que puede fomentarse la responsabilidad y autonomía por el aprendizaje.

Bajo estas consideraciones, en el aula de FLE es posible promover entonces prácticas de autorregulación que favorezcan un tratamiento del error. Esto se ve claro en Zimmerman (2000) al compartir que estas prácticas de autorregulación tienen un que ver con la autoevaluación, organización en el aprendizaje, establecimiento de metas, o la búsqueda de la información. Refiriendo específicamente a la autoevaluación, consideramos medular que el estudiante de FLE reconozca sus fortalezas y debilidades para poder determinar qué acciones deberá tomar en cuanto a su aprendizaje, además de construir y fomentar estrategias que le permitan cumplir con las metas establecidas.

Esto lleva sin duda alguna, a que un estudiante que analiza será capaz de ir más allá del contenido esperado o, en ciertos casos, resolver por su propia cuenta dudas que quizá en el aula no son resueltas. También, considerando que, por distintos factores, el estudiante prefiere omitir cualquier cuestionamiento y llevar con él esas dudas durante el aprendizaje. Desde la función de la autorregulación, Consef roy (2010), comparte que ésta sustenta un acercamiento a una motivación inicial en el estudiante, el establecimiento de objetivos, una capacidad de autoevaluarse y de auto observación.

En cuanto a la autorregulación se reconocen dos elementos principales: las estrategias de autorregulación que implementa el estudiante (el que es consciente de su aprendizaje), y las estrategias de enseñanza por parte del docente que integran la autorregulación como mediador en el aprendizaje (Bégin, 2005). Pues desde Usher y Schunk (2017) las estrategias no son innatas, sino que usualmente se aprende, lo mismo hacia el fomento de la autonomía.

Refiriendo a este acercamiento, Dunkan y McKeachi (2005) generalizan las siguientes estrategias de autorregulación: a) las estrategias cognitivas que favorecen el pensamiento crítico, b) las estrategias dirigidas a la planeación, monitoreo y regulación que destacan el aspecto metacognitivo, y por último, c) las estrategias que permiten el trabajo en equipo o la coevaluación. Aunado a ello, insistimos que la sustentación del tratamiento del error requiere de un compromiso dual. En principio, vemos necesario que se otorgue una responsabilidad al estudiante quien sea capaz de reconocer qué está bien y qué no desde la función de la producción de la lengua.
De esto surge nuestro interés de establecer que el error y su tratamiento es dependiente del cambio en la percepción del estudiante en su propio aprendizaje. Pero existe un rol medular: el del docente quien adecua las prácticas de aprendizaje desde la proyección de las metas del curso y de la retroalimentación que otorga. Hacia este expuesto vemos que, para hablar del tratamiento del error, los roles en el aula deberán redefinirse siendo un proceso conjunto (Zimmerman y Schunk, 2011). Por lo que el acompañamiento e integración de estas prácticas autorreguladoras dentro de la enseñanza y aprendizaje de FLE resultaría asertivo. Considerando que la intención principal es promover la motivación y la autonomía que permitirán que el estudiante tenga la iniciativa de mejorar en su aprendizaje (Wolters, 2003).

En general, queremos dejar claro que es posible dimensionar al error desde distintas posturas pero que hacia el tratamiento del error la autorregulación sugiere una ruta interesante para el aprendizaje. Por un lado, que el docente construya un escenario de aprendizaje basado en la reflexión, y en conjunto, de propiciar prácticas autoregulatorias que permitan al estudiante comprender sus necesidades en la lengua meta.

\section{Método}

En este estudio hacemos presente un enfoque mixto tras identificar la recurrencia de errores en el adjetivo desde lo cuantitativo, y realizamos un acercamiento a la interpretación cualitativa de los datos basados en el criterio etiológico y la tipología del error, recordando que este criterio busca brindar una explicación sobre las causas y el origen del error. Al tener esta aproximación se buscaba que la interpretación de los datos no sólo ubicara una identificación global sino dirigirnos a una interpretación más exhaustiva que permitiera un acercamiento al tratamiento del error basado en la autorregulación.

En particular desde la construcción del corpus auténtico de producciones escritas de FLE en el nivel A1 y A2 fue posible tener un entendimiento que diera validez a nuestro estudio. Hunston (2010) establece que el corpus lingüístico parte del procesamiento de datos específicos a partir de una metodología conveniente para el estudio que busca encontrar frecuencias y características del objeto por analizar. Para hacer esto posible se requiere que se definan los elementos que formarán parte de los datos que se procesarán y que partirán de un etiquetado específico. En cuanto al propósito principal de este trabajo dedicaremos los siguientes párrafos a describir el corpus de aprendices. Ferreira y Elejalde (2017) mencionan que la metodología basada en la lingüística de corpus de aprendices cobra relevancia en el campo de las lenguas. Las autoras precisan que este interés es a razón de entender a la lengua desde contextos reales donde el investigador puede acceder a colecciones orales o escritas. 
Nuestro análisis del error basado en corpus de aprendices reconoce un alcance descriptivo que fue posible desde y para los datos obtenidos en el estudio. La siguiente tabla sintetiza las fases generales que enmarcan este trabajo:

1. Conformación del corpus de estudiantes.

2. Reconocimiento y tipología del error.

3. Recomendaciones hacia le tratamiento del error desde las prácticas de autorregulación.

\section{Fase 1: conformación del corpus de estudiantes FLE}

En cuanto a esta primera fase, se partió de la recolección de producciones escritas de los niveles A1 y A2 provenientes de estudiantes de francés lengua extranjera adscritos a los cursos del centro de idiomas. Mismos, que llevan una formación específica hacia la especialización en el campo de la enseñanza de lenguas extranjeras. El criterio de selección, como se menciona, era dependiente del perfil de los participantes. Esto es importante porque el razonamiento de nuestro estudio desde la tipología, es que las lenguas próximas influyen de cierta manera en el desarrollo de la lengua meta: en este caso FLE. Por lo que destacamos el siguiente perfil de nuestros participantes:

1. Ser hispanohablante.

2. Contar con el inglés como lengua extranjera dentro de su formación profesional.

Bajo este criterio de selección, nuestro corpus de estudiantes tras su depuración, se compone de 23 actividades que nos otorgan 188 producciones escritas (PE) en el nivel A1. Además, se recopilaron 29 actividades correspondientes al nivel A2 que resultan en 171 textos procesados en total. Además añadimos que el proceso de recolección y conformación del corpus fue un proceso tardado pues no fue algo sistematizado, sino manual.

\section{Fase 2: reconocimiento y tipología del error.}

Al ser un estudio no automatizado, y tras dar lectura individ ual a los diversos textos partimos en una primera etapa hacia la identificación de errores desde un orden generalizado. El cual nos permitió posteriormente indagar e identificar en qué categorías gramaticales se reflejaba el error, en particular bajo este estudio el del adjetivo.

Tras el reconocimiento de errores fue posible integrar nuestra parrilla de análisis la cual consistió en la tipología del error anteriormente descrita. Basados en el error interlingual, el cual es producto de la presencia de otras lenguas: siendo en nuestro estudio el español e inglés. Por otra parte, los errores intralinguales que son producto de desviaciones o generalizaciones de normas y reglas en la lengua meta.

No olvidemos que esta tipología del error se basa en las intenciones del criterio etiológico. Alexopoulu (2006) comparte que esta etapa de explicación desde el criterio etiológico es desafiante para el investigador, pues demanda que sea capaz de identificar y reconocer las causas por las cuales se origina el error. Se justifica que esta tipología del error basada en este criterio permite un acercamiento conveniente para nuestro espacio formativo y que puede propiciar un tratamiento del error. También destacamos que la aportación que hacemos en este artículo, además del análisis del error en el adjetivo, es propiciar algunas reflexiones en cuanto a las prácticas autorreguladoras como parte de la evaluación y el tratamiento del error. Es así que, para la complejidad de este tipo de estudios y análisis no resulta factible una intervención inmediata pues los datos recuperados, que parten de nuestro corpus de estudiantes son extensos y se recopilaron durante un periodo específico.

\section{Análisis y discusión}

En relación con el objetivo de la investigación: reconocer la presencia de errores en el adjetivo y su concordancia desde el criterio etiológico, y tras haber precisado las fases del estudio partimos hacia la explicación de los datos obtenidos. Debido a las características de nuestro corpus socializaremos los más significativos y recurrentes dentro del corpus de FLE.

Compartimos que los datos que a continuación se enlistan se establecen bajo el sustento teórico que emana del Análisis de Errores, su criterio y su tipología. Esto se hace posible a partir de la discusión del error y su tratamiento, el cual exige un cambio en la interpretación y percepción del investigador. En este entendido se muestra la siguiente tabla que permite visualizar la frecuencia en la que se presentan los errores en el adjetivo por nivel de conocimiento de la lengua. Posteriormente, se enuncia una descripción del error en el adjetivo con los ejemplos más relevantes dentro del corpus.

\begin{tabular}{|l|l|l|l|l|}
\hline Nivel & $\begin{array}{l}\text { Producciones } \\
\text { escritas }\end{array}$ & $\begin{array}{l}\text { Errores } \\
\text { (Adj) }\end{array}$ & Interlingual & Intralingual \\
\hline A1 & 188 & 121 & 17 & 104 \\
\hline A2 & 171 & 92 & 10 & 82 \\
\hline
\end{tabular}

Tabla 1 El error en el adjetivo

Como se ha mencionado, el interés de este espacio se centra en la socialización de los errores en el adjetivo (Adj) más representativos para nuestro corpus. Tras proveer de una subclasificación del error a razón de la tipología de errores: interlinguales e intralinguales. En lo particular, reconociendo que en esta categoría hacemos alusión a los errores que refieren a la concordancia de género y número. A continuación, iniciamos con la presentación de los errores identificados en el adjetivo desde la concordancia entre género y número correspondientes al nivel A1.

\section{El error en el adjetivo nivel A1}

Precisamos que los resultados para esta investigación, refieren exclusivamente al adjetivo y su concordancia (género y número) independientemente de los errores correspondientes a 
otras categorías gramaticales que podrían identificarse en los distintos ejemplos.

\begin{tabular}{|c|c|c|}
\hline *La nourriture (nf) c'est délicieux & ADJ & Concordancia género \\
\hline *dans l'espoir de $\boldsymbol{t} \boldsymbol{u}$ reponse (nf) & ADJ & Concordancia género \\
\hline *Mon équipe (nf) préférée & ADJ & Concordancia género \\
\hline *De mon maison (nf) à l'école & ADJ & Concordancia género \\
\hline $\begin{array}{l}\text { *C'est pourquoije crois que la musique } \\
\text { (nf) est merveilleux }\end{array}$ & ADJ & Concordancia género \\
\hline $\begin{array}{l}\text { *j'attendais que la nourriture (nf) était } \\
\text { délicieux }\end{array}$ & ADJ & Concordancia género \\
\hline $\begin{array}{l}\text { *Elle (nf) a l'air gentil mais un peu } \\
\text { serieuse }\end{array}$ & ADJ & Concordancia género \\
\hline $\begin{array}{l}\text { *Physiquement, elle (nf) est haut, jolie, } \\
\text { sa silhouette est maigre et petit. }\end{array}$ & ADJ & Concordancia género \\
\hline *J'a ime la zone (nf) vert & ADJ & Concordancia género \\
\hline $\begin{array}{l}\text { *Au futur, probablement les portables } \\
\text { (nmpl) seront plus différent }\end{array}$ & ADJ & Concordancia número \\
\hline $\begin{array}{l}\text { *Le nouveau portables (nmpl) sont } \\
\text { plus petites. }\end{array}$ & ADJ & $\begin{array}{l}\text { Concordancia } \\
\text { género/número }\end{array}$ \\
\hline $\begin{array}{l}\text { *Je vais continuer avec la lecture de } \\
\text { cette livre }(\mathbf{n m}) \text { pendant la semaine. }\end{array}$ & ADJ & Concordancia género \\
\hline $\begin{array}{l}\text { *parler un moment avec sont famille } \\
\text { (nf) et son amis (nmpl). }\end{array}$ & ADJ & $\begin{array}{l}\text { Concordancia género } \\
\text { Concordancia número }\end{array}$ \\
\hline $\begin{array}{l}\text { *Nous sortons à quelques lieux (nmpl) } \\
\text { ensemble et elles sont très amusant }\end{array}$ & ADJ & Concordancia número \\
\hline *L'ours (nmpl) grizzle sont solitaire. & ADJ & Concordancia número \\
\hline *J'adore le sourire de mon fille (nf). & ADJ & Concordancia género \\
\hline *c'est une femme (nf) intelligent. & ADJ & Concordancia género \\
\hline $\begin{array}{l}\text { *j'aime un petite lieux (nmpl) de } \\
\text { norriture }\end{array}$ & ADJ & Concordancia número \\
\hline *Mirandela (nf-ville) n'est trop chaud & ADJ & Concordancia género \\
\hline $\begin{array}{l}\text { *La mademoiselle (nf) de la maison est } \\
\text { tombé amoureux }\end{array}$ & ADJ & Concordancia género \\
\hline $\begin{array}{l}\text { *nous avons trouvé une belle ville (nf) } \\
\text { qui est très intéressant }\end{array}$ & ADJ & Concordancia género \\
\hline $\begin{array}{l}\text { *Je n'adore pas faire de petit choses } \\
\text { (nf) }\end{array}$ & ADJ & Concordancia género \\
\hline
\end{tabular}

Tabla 2 El error en el adjetivo Al

Tal como ilustran los ejemplos existen errores asociados al género, es decir, el estudiante requiere ser consciente del sentido que expresa: entre lo masculino (livre, équipe) o femenino (ville, mademoiselle,femme). Entendemos que esta posición tiene relación con la ausencia de distinción en las lenguas dentro de su repertorio lingüístico. Siendo que en español no se hace esta distinción: un hombre inteligente/una mujer inteligente. Lo que contempla también en muchos casos otro factor determinante, el hecho, de omitir la $e$ como terminación del adjetivo (intelligent/intelligente) recurso del error que se define como generalizaciones.

Otras evidencias abordan una relación entre el adjetivo posesivo (mon, ma, mes), pero también hacia el demostrativo (ce,cette,cet,ces). Es interesante ver este tipo de elementos presentes en un corpus, pues resulta la evidencia de un aprendizaje en tránsito. Pues son contenidos que se ven en las primeras lecciones del curso y que se tratan a lo largo de las diversas unidades temáticas. No obstante, es en los primeros niveles que por naturaleza el error se hará presente pero el cuestionamiento es, ¿qué ocurre al no hacer un tratamiento del error? Esta falta de conciencia metalingüística también refleja un comportamiento a la concordancia con el número. Donde simplificamos este expuesto que lo singular y/o plural condicionan la esencia del adjetivo. Es, de tal forma, que dentro de los datos recuperados encontramos que el estudiante hace omisiones y generalizaciones en cuanto al sustantivo más hacia la tipología intralingual.

\section{El error en el adjetivo nivel A2}

Como se dijo líneas arriba, sostenemos que el error estará presente dentro del aprendizaje; eso es indiscutible. Sin embargo, detallamos que los errores requieren de un tratamiento inicial pues el estudiante tiende a llevar consigo esos comportamientos de la lengua hacia nuevos niveles de aprendizaje. Aquí se toma a consideración, que también transita a un nivel que demanda el aprendizaje de otros elementos lingüísticos que deberá concientizar. Ahora revisemos los datos que refieren al nivel A2 que integra nuestro corpus de estudiantes de FLE.

\begin{tabular}{|c|c|c|}
\hline $\begin{array}{l}\text { Jusque cet moment (nm) je n'avais pas } \\
\text { travaillé }\end{array}$ & ADJ & $\begin{array}{l}\text { Concordancia } \\
\text { género }\end{array}$ \\
\hline $\begin{array}{l}\text { ou vivre seul et tranquile et ça c'est } \\
\text { genial*(la femme-nf) }\end{array}$ & ADJ & $\begin{array}{l}\text { Concordancia } \\
\text { género }\end{array}$ \\
\hline $\begin{array}{l}\text { Géométrique, spacieux, lumineux, mobilier } \\
\text { durable (la cuisine-nf) }\end{array}$ & ADJ & $\begin{array}{l}\text { Concordancia } \\
\text { género }\end{array}$ \\
\hline $\begin{array}{l}\text { avec touts les enfants (nmpl) qui habitaient } \\
\text { dans le même rue }\end{array}$ & ADJ & $\begin{array}{l}\text { Concordancia } \\
\text { número }\end{array}$ \\
\hline l'eau (nf) a été froid. & ADJ & $\begin{array}{l}\text { Concordancia } \\
\text { género }\end{array}$ \\
\hline $\begin{array}{l}\text { elle a été une personne (nf) trés important } \\
\text { dans ma vie }\end{array}$ & ADJ & $\begin{array}{l}\text { Concordancia } \\
\text { género }\end{array}$ \\
\hline $\begin{array}{l}\text { Elle est une personne (nf) très important } \\
\text { pour moi }\end{array}$ & ADJ & $\begin{array}{l}\text { Concordancia } \\
\text { género }\end{array}$ \\
\hline $\begin{array}{l}\text { David (nm) est une personne très tranquille } \\
\text { et intelligente }\end{array}$ & ADJ & $\begin{array}{l}\text { Concordancia } \\
\text { género }\end{array}$ \\
\hline $\begin{array}{l}\text { Eduardo }(\mathbf{n m}) \text { est une homme patiente, } \\
\text { généreux, très intelligente y pasionnante }\end{array}$ & ADJ & $\begin{array}{l}\text { Concordancia } \\
\text { género }\end{array}$ \\
\hline Elle a été ma tante (nf) elle est mort & ADJ & $\begin{array}{l}\text { Concordancia } \\
\text { género }\end{array}$ \\
\hline Il (nm) est $m a$ meilleure a mi & ADJ & $\begin{array}{l}\text { Concordancia } \\
\text { género }\end{array}$ \\
\hline $\begin{array}{l}\text { la grand personne (nf) que je veux rendre } \\
\text { hommage }\end{array}$ & ADJ & $\begin{array}{l}\text { Concordancia } \\
\text { género* }\end{array}$ \\
\hline Je suis desolé pour ton situation (nf) & ADJ & $\begin{array}{l}\text { Concordancia } \\
\text { género }\end{array}$ \\
\hline éviter la chirurgie, et qu'il (nm) est belle & ADJ & $\begin{array}{l}\text { Concordancia } \\
\text { género }\end{array}$ \\
\hline la vie et les personnes (nf) importants & ADJ & $\begin{array}{l}\text { Concordancia } \\
\text { género }\end{array}$ \\
\hline Elle venait de voir son fille (nf). & ADJ & $\begin{array}{l}\text { Concordancia } \\
\text { género }\end{array}$ \\
\hline $\begin{array}{l}\text { Je pense que la nationalité (nf) n'est pas } \\
\text { important. }\end{array}$ & ADJ & $\begin{array}{l}\text { Concordancia } \\
\text { género }\end{array}$ \\
\hline la chose (nf) plus important & ADJ & $\begin{array}{l}\text { Concordancia } \\
\text { género }\end{array}$ \\
\hline
\end{tabular}

Tabla 3 El error en el adjetivo A2

Como muestra de los resultados correspondientes al nivel A2, presentamos que la concordancia de género y número es un elemento presente aún en un nivel superior al anteriormente 
descrito. Vemos que el error, desde el criterio y tipología considerada, se hace notar a través de los diversos ejemplos presentados:

\section{Elle venait de voir son fille ( $n f)$. \\ Jusque cet moment (nm) je n'avais pas travaillé}

Por un lado, desde el reconocimiento que tiene la lengua próxima desde el repertorio lingüístico del estudiante al pensar en similitudes hacia el uso de los adjetivos en la lengua meta. Y por otra, desde la falta de comprensión que deriva de omisiones y generalizaciones del adjetivo ausente de una concordancia (David (nm) est une personne très tranquille et intelligente). En muchos casos, también consideramos que en este nivel puede ser a razón de otros factores extralingüísticos: es decir distracciones o momentos de falta de concentración por parte del estudiante. Esto presente cuando el estudiante ya en un nivel A1 es capaz de distinguir los distintos pronombres ( $j e$, tu, il/elle, nous, vous, ils/elles).

Para cerrar este apartado, pasamos a mencionar que en evidencia de los datos obtenidos encontramos que algunos refieren a los adjetivos demostrativos que pueden generar un conflicto de generalizaciones para el estudiante hispanohablante por sus variaciones: $c e$, cette, cet, ces. Otros casos, al no seguir un orden sobre el sujeto o sustantivo el cual forma parte de la oración (femme-nf, cuisine-nf). Además de aquellos casos que contempla la necesidad de fomentar una concientización de número.

\section{Recomendaciones hacia le tratamiento del error desde las prácticas de autorregulación.}

Como parte de la tercera etapa, y desde el análisis basado en un extracto de nuestro corpus de estudiantes de Francés Lengua Extranjera, destacamos los siguientes puntos. En primer lugar, sugerimos al docente de lenguas que los estudios que consideran el Análisis de Errores partan de una estructura específica y con una intención definida. De inicio, se requiere partir de la conformación del corpus de aprendices (oral o escrito) que demandará tiempo para el investigador pero que brinda total autenticid ad para este tipo de estudios.

En este análisis también reconocemos la importancia que tiene la identificación y clasificación del error que, independientemente de ser un proceso complejo, requiere de una revisión exhaustiva pues son diversos los datos que podrán recuperarse. Pues la clasificación que se otorgue depende del investigador ya que encontramos que pudiera clasificarse el error de una u otra forma: de lo morfológico o morfosintáctico por mencionar. En definitiva, sugerimos que antes de cualquier descripción y explicación del error primero deberá entenderse con claridad el contexto de donde surge el corpus, así será posible determinar qué tipología otorgar al error.

En relación con las aportaciones específicas del estudio, reiteramos que la conformación de un corpus lingüístico nos muestra un acercamiento único al contexto, donde es posible encontrar incidencias en el aprendizaje que debe revalorar el profesorado. En cuanto a la tipología del error es cierto que existen distintas formas de comprender el error, pero se debe emplear un criterio a razón de las necesidades del ambiente de aprendizaje. Fue en nuestro estudio que partimos del criterio etiológico basado en la descripción y explicación de errores porque no sólo buscábamos etiquetar el error. Dentro estas aportaciones, queremos dejar claro que el error debe entenderse desde la función que puede tener para el estudiante, quien debe ser capaz de razonar el error y autorregularse.

Basado en el análisis dejamos en evidencia a su vez las siguientes consideraciones. En los ejemplos que compartimos correspondientes al error en el adjetivo vemos notables incidencias en cuanto a la concordancia de género y número. Se presentan errores frecuentes que parten de asociaciones con el repertorio lingüístico del estudiante, pero también aquellos relacionados con desviaciones y generalizaciones, todo derivado de la falta de concientización metalingüística.

A ello, dirigimos la mirada al hecho que sí es importante partir que el tratamiento del error es dependiente del contexto, por ello se insiste en recomendar la conformación de un corpus de estudiantes (oral o escrito). También se podría afirmar que el error demanda de una redefinición de la evaluación que puede sugerir más que una calificación, se trata desde luego de proponer líneas específicas que permitan un tratamiento del error.

Como última recomendación dentro del tratamiento del error, consideramos integrar a la autorregulación como parte del proceso de aprendizaje. Es importante anotar que su integración favorecerá que el estudiante determine cuáles son las necesidades específicas que tiene hacia la lengua y proyecte metas específicas. Esto se logra al momento de otorgarle una evaluación, pero sobre todo una corrección que le ayude a entender qué requerirá para mejorar su competencia en la lengua. Este elemento, hasta cierto punto, resulta complejo pues un feedback puede resultar en un recurso temporal pero que no provee una concientización de la lengua meta. Nosotros referimos que una vez identificado los errores se proponga una autorregulación integrada dentro del proceso de enseñanza y aprendizaje a través de los contenidos del curso. Esto desde Pintrich (1990) y más reciente Cosnefroy (2011), tras integrar modelos de autorregulación que sugieren: planeación, monitoreo, control/revisión, reacción y reflexión.

\section{Conclusión}

Como cierre de este trabajo, es evidente que el análisis de errores forma parte del quehacer docente, tras identificar como parte de la evaluación cuál es el desempeño del estudiante en la producción de la lengua meta. Es en esta identificación que se pone de manifiesto que el error resulta el punto de partid a para la implementación de diversas acciones que favorezcan el 
aprendizaje. A nuestro criterio, consideramos que la conciencia metalingüística requiere de un compromiso por parte del estudiante de lengua quien deberá entender y razonar las representaciones lingüísticas de la lengua meta. Es decir, de ser capaz de identificar reglas y normas de la lengua que favorezcan su aprendizaje. Sobre todo en nuestro contexto de formación de formadores en el campo de las lenguas extranjeras.

Como se afirma en apartados anteriores, la comprensión del conocimiento lingüístico es dependiente de las acciones (docente y estudiante) y el posible tratamiento que se implemente en el aula. Por tanto, consideramos que el fomentar la autorregulación como parte del aprendizaje de una lengua meta es un recurso valioso que permitirá poner en perspectiva el estado actual del estudiante en su aprendizaje. En consecuencia, al hablar de y tratar estas nociones, es posible fomentar una concientización por el aprendizaje y sobre todo de dar un valor a la autoevaluación. De esta forma el estudiante será capaz de reflexionar que requerirá para desarrollar la lengua y será un estudiante autónomo dentro y fuera del aula.

\section{REFERENCIAS}

[1] Alba Quiñones, V. de (2009). El análisis de errores en el campo del español como lengua extranjera: algunas cuestiones metodológicas. Revista Nebrija de Lingüística Aplicada, 5 (3), 1-16

[2] Alexopoulou, A. (2006). Los criterios descriptivo y etiológico en la clasificación de los errores del hablante no nativo: una nueva perspectiva. Porta Linguarum: revista internacional de didáctica de las lenguas extranjeras, (5), 17-36.

[3] Aregu, B. B. (2013). Enhancing Self-Regulated Learning in Teaching Spoken Communication: Does It Affect Speaking Efficacy and Performance? Electronic Journal of Foreign Language Teaching, 10(1).

[4] Baralo, M. (1999). La adquisición del español como lengua extranjera. Arco/Libros.

[5] Bégin, C. (2008). Les stratégies d'apprentissage: un cadre de référence simplifié. Revue des sciences de l'éducation, 34 (1), 47-67. https://doi.org/10.7202/018989ar

[6] Besse, H., y Porquier, R. (1984). Grammaires et didactique des langues (Vol. 9). Hatier.

[7] Brown, H. (2006). Principles of Language Learning and Teaching.

[8] Cenoz, J. (2001). The effect of linguistic distance, L2 status and age on cross-linguistic influence in third language acquisition. Cross-linguistic influence in third language acquisition: Psycholinguistic perspectives, $111(45), 8-20$.

[9] Cosnefroy, L. (2011). L'apprentissage autorégulé: entre cognition et motivation. Presses universitaires de Grenoble

[10] Corder, S. P. (1983). A role for the mother tongue. Language transfer in language learning, 1, 85-97.
[11] De Angelis, G., y Selinker, L. (2001). Interlanguage transfer and competing linguistic systems in the multilingual mind. Bilingual Education and Bilingualism, 42-58.

[12] Dewaele, J. M. (1998). Lexical inventions: French interlanguage as L2 versus L3. Applied linguistics, 19(4), 471-490.

[13] Duncan, T. y McKeachie, W. (2005) The Making of the Motivated Strategies for Learning Questionnaire. Educational Psychologist, 40(2), 117-128.doi: 10.1207/s15326985ep4002_6

[14] Ellis, R. (1985). Understanding second language acquisition (Vol. 47, p.73). Oxford: Oxford university press.

[15] Fernández, S. (1997). Interlengua y análisis de errores: en el aprendizaje del español como lengua extranjera. Edelsa Grupo Didascalia.

[16] Gómez, J. y Ferreira, A. (2017). Análisis de errores recurrentes en el Corpus de Aprendices de Español como Lengua Extranjera, CAELE. Revista Brasileira de Linguística Aplicada, 17(3), 509-538. Epub July 27, 2017.https://doi.org/10.1590/1984-6398201710927

[17] Granger, S. (2009). The contribution of learner corpora to second language acquisition and foreign language teaching. Corpora and language teaching, 33(13).

[18] Griffin, K. (2005). Lingüística aplicada a la enseñanza del español como 2/L. Madrid: Arco/Libros.

[19] Hammarberg, B. (2001). Roles of Ll and L2 in L3 production and acquisition. Cross-linguistic influence in third language acquisition: Psycholinguistic perspectives, 31, 21.

[20] Han, Z., y Odlin, T. (Eds.). (2006). Studies of fossilization in second language acquisition (Vol. 14). Multilingual Matters.

[21] Lantolf, J. P. (2006). Language emergence: Implications for applied linguistic. A sociocultural perspective. Applied linguistics, 27(4), 717728 .

[22] Nemser, W. (1971). Approximative systems of foreign language learners. IRAL-International Review of Applied Linguistics in Language Teaching, 9(2), 115-124.

[22] Pendar, N., y Chapelle, C. A. (2008). Investigating the promise of learner corpora: Methodological issues. CALICO journal, 25(2), 189.

[23] Pintrich, P.R. (2000). The Role of Goal Orientation in Self-Regulated Learning. In M. Boekaerts, P. R. Pintrich, \& M. Zeidner (eds.), Handbook of Self-Regulation (pp. 451-502). Academic Press. doi:https://doi.org/10.1016/B978-012109890-2/50043-3

[24] Porquier, R. (1977). L'analyse des erreurs. Problèmes et perspectives. Études de linguistique appliquée, 25, 23.

[25] Selinker, L. (1972). Interlanguage. Error analysis. Perspectives on Second Language Acquisition. London: Longman.

[26] Touchie, H. Y. (1986). Second language learning errors: Their types, causes, and treatment. JALT journal, 8(1), 75-80.

[27] Usher, E. L., y Schunk, D. H. (2017). Handbook of Self-Regulation of Learning and Performance. DH Schunk \& JA Greene, Social Cognitive Theoretical Perspective of Self-Regulation, 19-35.

[28] Vázquez, G. E. (1999). ¿ Errores?i Sin falta!. Edelsa Grupo Didascalia. 
Biannual Publication, Revista Lengua y Cultura, Vol. 2, No. 4 (2021) 46-55

[29] Zimmerman, B. y Schunk, D. H. (2011). Handbook of self-regulation of learning and performance. Routledge.

[30] Zimmerman, B. (2000). Attaining Self-Regulation: a social cognitive perspective. En M. Z. En M. Boekaerts, Handbook of self regulation (pp. 13-39). Academic Press. http://dx.doi.org/10.1016/B978012109890-2/50031-7. 